\title{
Flexión de Una Barra Delgada Empotrada en un Extremo: Aproximación para Pequeñas Pendientes
}

\author{
Tarsicio Beléndez ${ }^{1}$, Cristian Neipp ${ }^{2}$ y Augusto Beléndez ${ }^{2}$ \\ ${ }^{1}$ Departamento de Ciencia y Tecnología de los Materiales \\ Universidad Miguel Hernández de Elche \\ Avda. del Ferrocarril, s/n. E-03202, Elche (Alicante), España \\ ${ }^{2}$ Departamento de Física, Ingeniería de Sistemas y Teoría de la Señal \\ Universidad de Alicante, Apartado 99, E-03080 Alicante, España
}

Recebido em 7 de junho, 2002. Aceito em 7 de setembro, 2002.

\begin{abstract}
En los cursos de Física y Mecánica para estudiantes universitarios de Ciencias e Ingeniería, y dentro del tema dedicado a la elasticidad, se estudian los sólidos deformables, así como los fenómenos de tracción, compresión, cizalladura, torsión, flexión y pandeo. En el caso de la flexión uno de los ejemplos que suele analizarse es el de una barra delgada empotrada en un extremo sobre la que actúa una fuerza concentrada vertical en el extremo libre. El estudio se hace para pequeños desplazamientos o pequeñas pendientes de la barra, lo que permite integrar fácilmente la ecuación diferencial de la barra flexionada y calcular la elástica y la flecha o desplazamiento vertical del extremo libre. Sin embargo, la aproximación para pequeñas pendientes que se hace en la bibliografía es incompatible con otra consideración que siempre se tiene en cuenta y es que la longitud de la fibra neutra de la barra no cambia cuando la barra flexiona. En este trabajo se presenta, para al problema de la flexión de una barra delgada en voladizo, una aproximación para pequeños desplazamientos o pequeñas pendientes de la barra "menos aproximada de lo común". Para ello se introduce como hipótesis la constancia de la longitud de la fibra neutra de la barra lo que permite obtener unas ecuaciones más precisas que las que aparecen en la bibliografía. Los resultados obtenidos se han comparado con los exactos así como con las medidas experimentales realizadas en el laboratorio con una regla de acero empotrada en un extremo. Las ecuaciones de la elástica se obtienen siguiendo un tratamiento análogo al desarrollado por Feynman cuando estudia la viga flexionada en su libro de Física, por lo que el desarrollo aquí presentado puede ser seguido sin muchos problemas por cualquier estudiante de Física o Mecánica de los primeros cursos universitarios.
\end{abstract}

\section{Introducción}

Las vigas generalmente son cuerpos sólidos de forma alargada y sección recta constante, de gran interés en ingeniería y arquitectura, que normalmente se utilizan en posición horizontal y siendo su longitud grande comparada con las dimensiones de su sección recta. Las vigas pueden estar sometidas a cargas concentradas, cargas distribuidas, o a pares (momentos concentrados) que actúen solos o en una combinación cualquiera, siendo la flexión la principal deformación que sufren [1].

Puede definirse una viga como un sólido homogéneo e isótropo engendrado por una sección transversal, que generalmente admite un plano de simetría y cuyo centro de gravedad describe una curva o línea, denominada directriz, siendo el plano que contiene a la sección transversal normal a dicha directriz [2]. Cuando una viga flexiona debido a la presencia de fuerzas exteriores, existen algunas partes de la viga que se acortan debido a que están sometidas a una compresión, y, sin embargo, otras zonas se alargan por efecto del momento flector que hace que estén sometridas a tracción. Pero hay una línea en la viga en la que las fibras no se acortan ni se alargan, es decir, que no están sometidas a tensión. Esta línea se denomina línea neutra o fibra neutra (Fig. 1) [2,3]. En la fibra neutra se encuentra el centro de gravedad de la sección transversal; por tanto, para conocer la localización de dicha fibra basta con calcular el centro de gravedad de la sección transversal de la viga.

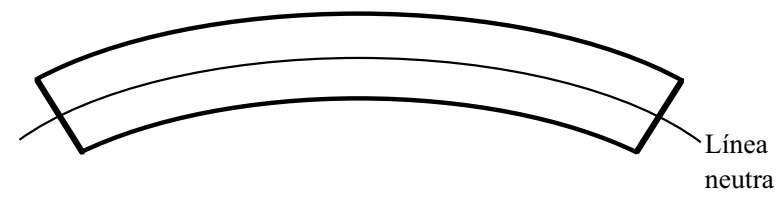

Figura 1. Fibra neutra de una viga flexionada.

Un caso particular de vigas que se estudian en los 
primeros cursos universitarios de Física y Mecánica de las titulaciones de Ingeniería y Arquitectura e incluso en la asignatura de Mecánica de Bachillerato son las estáticamente determinadas o isostáticas [1-3], para las que se pueden obtener las reacciones en los apoyos a partir de las ecuaciones de la Estática, es decir, imponiendo las condiciones de que la suma de fuerzas sea nula y la suma de momentos respecto a un punto también lo sea. El estudio de la flexión de las vigas isostáticas puede encontrarse en algunos textos de Física General $[2,4,5]$, Mecánica (en su parte de Estática) [1,6] y Mecánica de Materiales [7,8]. Asimismo, las vigas en voladizo, es decir, aquellas que se encuentran empotradas en un extremo y libres en el otro, han recibido recientemente atención en revistas en las que se publican trabajos relacionados con la enseñanza de la Física a nivel universitario [9-12]. Sin embargo, para el análisis que vamos a considerar en este trabajo basta con tomar como punto de partida el estudio de la viga flexionada para pequeñas pendientes que hace Feynman [5], al ser bastante claro (como no podía ser de otra manera) y suficiente para comprender el comportamiento de estos elementos constructivos cuando se someten a acciones externas.

En el presente trabajo se va a analizar la flexión de una barra delgada en voladizo en el caso particular en el que los desplazamientos de la barra son pequeños. Esto da lugar a que las pendientes de la curva elástica de la barra sean pequeñas, lo que permite hacer una serie de aproximaciones que dan lugar a una ecuación diferencial lineal de segundo orden fácilmente integrable. Sin embargo, en este trabajo se va a comprobar como es posible obtener unos resultados menos aproximados y más acordes con la realidad física de la barra flexionada que los que suelen presentarse en la bibliografía $[2,5,7,8]$. En particular, una cuestión que siempre se considera al estudiar la flexión de este tipo de elementos es que las deformaciones de la barra son pequeñas lo cual implica que, aunque la barra se desplace respecto a la horizontal, su longitud o más estrictamente la de su fibra neutra, permanece constante. Ésta es una cuestión muy importante que no suele tenerse en cuenta en los libros de texto de los primeros cursos universitarios pues el desarrollo que hacen, incluido el libro de Feynman, no tiene en cuenta que la longitud de la barra no cambia y de hecho las ecuaciones que presentan son incompatibles con la constancia de dicha longitud ya que la coordenada horizontal del extremo libre de la barra consideran que es igual a la longitud de la barra.

Se van a obtener distintas características de la barra delgada empotrada en un extremo: los desplazamientos máximos horizontal y vertical del extremo libre de la barra en función de la fuerza aplicada, y la curva que adopta la barra flexionada y que se denomina elástica [2-5]. Tanto los desplazamientos máximos como la elástica dependen del material y de la geometría de la barra [2, 3]. Se mostrarán algunos resultados numéricos y se compararán con los resultados que se obtienen haciendo uso de la aproximación para pequeñas pendientes que se hace en la bibliografía, con los correspondientes al caso exacto (sin ninguna aproximación), así como con los resultados experimentales obtenidos en el laboratorio utilizando como barra delgada una regla de acero.

\section{Ecuación diferencial de la elástica para pequeñas pendi- entes de la barra}

Considérese una barra delgada sometida a flexión de longitud $L$ y sección rectangular constante de base $b$ y altura $h$, empotrada en un extremo y sometida a una fuerza vertical $F$ en el extremo libre, tal y como se ve en la Fig. 2. Se supone que el material de la barra es elástico lineal, que su longitud es mucho mayor que las dimensiones laterales de la misma, que la longitud de la barra no cambia (estrictamente hablando la longitud de su línea neutra [8]) y que las deformaciones son pequeñas. Bajo estas hipótesis podemos utilizar la relación de Euler-Bernoulli entre el momento flector $M$ de la fuerza aplicada y el radio de curvatura $\rho$ de la barra deformada [5]:

$$
M=\frac{E I}{\rho}
$$

donde $E$ es el modulo de Young del material, $I$ es el momento de inercia de la sección transversal de la barra y $\rho$ es el radio de curvatura. El producto $E I$, que depende del tipo de material empleado y de las características geométricas de la sección de la barra, recibe el nombre de "módulo de rigidez a la flexión" de la barra o simplemente "rigidez"[7].

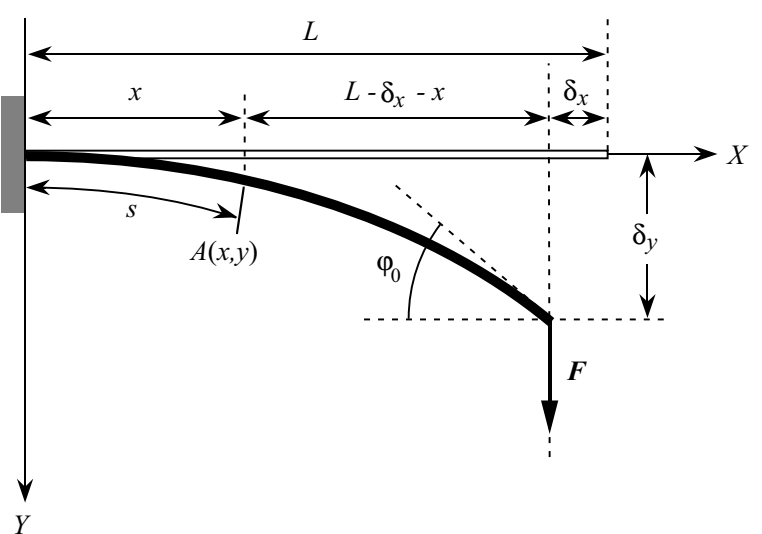

Figura 2. Barra delgada empotrada en un extremo y con una fuerza vertical concentrada en el extremo libre. Definición de parámetros.

El radio de curvatura $\rho$ de una curva de ecuación $y=y(x)$ puede calcularse mediante la ecuación $[2,5]$ :

$$
\frac{1}{\rho}=\frac{d^{2} y / d x^{2}}{\left[1+(d y / d x)^{2}\right]^{3 / 2}}
$$


En el caso particular en que el desplazamiento de los puntos de la barra flexionada respecto a los de la barra sin flexionar es pequeño, es decir, en el caso de pendientes pequeñas, es posible despreciar el término $(d y / d x)^{2}$ frente a la unidad en la ecuación (2), por lo que el radio de curvatura se puede calcular mediante la expresión:

$$
\frac{1}{\rho}=\frac{d^{2} y}{d x^{2}}
$$

lo que permite escribir la ecuación diferencial de la curva elástica para una barra delgada de material elástico lineal bajo la hipótesis de pequeños desplazamientos (elástica de pequeñas pendientes) en la forma:

$$
\frac{d^{2} y}{d x^{2}}=\frac{M}{E I}
$$

que es una ecuación diferencial lineal de segundo orden. Hasta aquí no hay ninguna diferencia a como se trata el problema en todos los libros de los cursos universitarios de Física y Mecánica [2, 5, 8]. La modificación que se va a introducir en este trabajo está relacionada con el cálculo del momento flector $M$ y está basada en el hecho de que la barra es inextensible (su fibra neutra), lo que implica que longitud de la barra no cambia debido a la flexión.

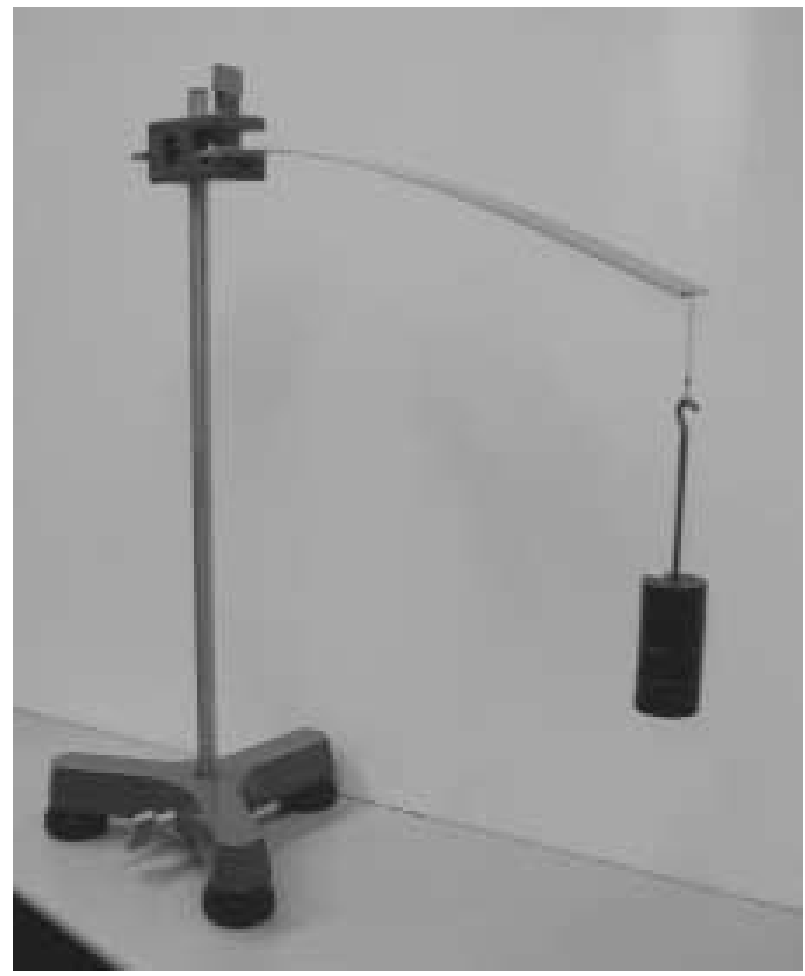

Figura 3. Fotografía de una barra delgada en voladizo.

Para obtener la ecuación de la curva elástica $y=$ $y(x)$ para pequeños desplazamientos de la barra hay que integrar la ecuación diferencial (4) y para ello es necesario conocer el momento flector $M$. La Fig. 3 muestra una fotografía de una barra delgada de longitud $L$ empotrada en un extremo sobre la que se aplica una fuerza vertical concentrada $F$ en el extremo libre. Puesto que la longitud de la barra deformada es también $L$, el extremo libre de la barra sufre tanto un desplazamiento vertical $\delta_{y}$ como uno horizontal $\delta_{x}$. Normalmente, y tal y como aparece en el libro de Feynman [5] y en otros textos [2, 4, 8], se supone que el desplazamiento horizontal $\delta_{x}$ es nulo, lo que no es compatible con el hecho de que la longitud de la barra no cambia tras la deformación. El momento flector $M$ debido a la carga puntual Faplicada en el extremo de la barra respecto a la sección situada a una distancia $x$ del empotramiento viene dado por la ecuación (Fig. 2):

$$
M(x)=F\left(L-\delta_{x}-x\right)
$$

y sustituyendo la ecuación (5) en la ecuación diferencial (4) de la elástica para pequeños desplazamientos (pequeñas pendientes), ésta toma la forma:

$$
\frac{d^{2} y}{d x^{2}}=\frac{F}{E I}\left(L-\delta_{x}-x\right)
$$

donde para la barra en voladizo las condiciones de contorno son:

$$
\begin{gathered}
y(0)=0 \\
\left(\frac{d y}{d x}\right)_{x=0}=0
\end{gathered}
$$

Con ayuda de las ecuaciones (7) y (8) es posible integrar fácilmente la ecuación (6), obteniéndose:

$$
\begin{aligned}
\frac{d y}{d x} & =\frac{F}{2 E I}\left[2\left(L-\delta_{x}\right) x-x^{2}\right] \\
y(x) & =\frac{F}{6 E I}\left[3\left(L-\delta_{x}\right) x^{2}-x^{3}\right]
\end{aligned}
$$

La ecuación (10) permite calcular el desplazamiento vertical máximo de la barra, $\delta_{y}$, el cual se presenta en el extremo libre de la misma. Su valor se obtiene, como se deduce de la Fig. 2, sustituyendo $x=L-\delta_{x}$ en la ecuación (10):

$$
\delta_{y}=\frac{F}{3 E I}\left(L-\delta_{x}\right)^{3}
$$

La ecuación (9) proporciona el valor de la pendiente de la elástica para cada valor de la coordenada $x$, mientras que la ecuación (10) es la ecuación cartesiana $y=y(x)$ de la elástica de la barra. Es importante señalar que la ecuación de la elástica (10) es válida siempre y cuando el cuadrado de la pendiente de la elástica sea mucho menor que la unidad, pues esa ha sido la hipótesis para obtener la ecuación (4), es decir, debe cumplirse la condición:

$$
\left(\frac{d y}{d x}\right)^{2}<<\quad 1
$$


Como la máxima pendiente tiene lugar en el extremo libre de la barra, es decir, para el punto de abcisa $x=$ $L-\delta_{x}$, teniendo en cuenta la ecuación (9) evaluada en ese punto, la condición de validez de las ecuaciones para pequeñas pendientes es:

$$
\left(\frac{F}{2 E I}\right)^{2}\left(L-\delta_{x}\right)^{4}<<1
$$

Para saber si en un caso práctico es o no aplicable la aproximación de pequeñas pendientes habría que verificar la condición (13). Sin embargo, en ocasiones es posible que se desconozca el valor del módulo de Young del material, $E$, pues lo que se pretende con el estudio experimental quizás sea su determinación. En este caso es fácil escribir la ecuación (13) sin que aparezca el valor de EI. Para ello se despeja EI de la ecuación (11) y se sustituye en la ecuación (13), obtieniéndose la condición de aplicabilidad de las ecuaciones para pequeñas pendientes en función de los desplazamientos horizontal y vertical del extremo libre, $\delta_{x}$ y $\delta_{y}$, respectivamente, los cuales pueden medirse fácilmente en el laboratorio. De la ecuación (13) se obtiene:

$$
\frac{9 \delta_{y}^{2}}{4\left(L-\delta_{x}\right)^{2}}<<1
$$

Sin embargo, para evaluar las ecuaciones (9)-(11) no sólo es necesario conocer el módulo de Young, $E$, el momento de inercia, $I$, y la fuerza aplicada, $F$, sino también el desplazamiento horizontal, $\delta_{x}$, del extremo libre de la barra, y éste es una incógnita del problema, pues a su vez es función de $E, I$ y $F$.Es aquí donde se hace uso de la hipótesis de que la barra es inextensible y, por tanto, su longitud $L$ no cambia (estrictamente hablando, la longitud de su fibra neutra, aunque si la barra es delgada se puede decir que ambas coinciden). Para introducir esta nueva hipótesis basta tener en cuenta la relación que existe entre el elemento de arco $d s$ y sus componentes cartesianas $d x$ y $d y$ :

$$
d s=\sqrt{d x^{2}+d y^{2}}=\sqrt{1+\left(\frac{d y}{d x}\right)^{2}} d x
$$

por lo que la longitud $L$ de la barra será:

$$
L=\int_{0}^{L-\delta_{x}} \sqrt{1+\left(\frac{d y}{d x}\right)^{2}} d x
$$

donde el límite superior de integración tiene en cuenta el hecho de que el valor máximo de la coordenada $x$ de la elástica de la barra no es $L$, como se indica en la bibliografía para el caso de la aproximación de pequeñas pendientes, sino $L-\delta_{x}$, como se ve en la Fig. 2. Sustituyendo la ecuación (9) en la ecuación (16), se obtiene:

$$
L=\int_{0}^{L-\delta_{x}} \sqrt{1+\left(\frac{F}{2 E I}\right)^{2}\left[2\left(L-\delta_{x}\right) x-x^{2}\right]^{2}} d x
$$

La ecuación (17) permite calcular el valor del desplazamiento horizontal del extremo libre de la barra cuando se conocen los valores de $L, E, I$ y $F$. Una vez obtenido el valor de $\delta_{x}$ ya es posible determinar el resto de parámetros que caracterizan la barra flexionada, en particular $\delta_{y}$ y la ecuación cartesiana de la elástica. Si se toma $\delta_{x}=0$ en las ecuaciones anteriores se obtienen los valores de $y(x)$ y $\delta_{y}$ siguientes:

$$
\begin{gathered}
y(x)=\frac{F}{6 E I}\left(3 L x^{2}-x^{3}\right) \\
\delta_{y}=\frac{F L^{3}}{3 E I}
\end{gathered}
$$

que son las que aparecen en al bibliografía [5, 9-12].

\section{Resultados numéricos}

Para comparar los resultados que se obtienen con la aproximación para pequeñas pendientes que se ha presentado en este trabajo con la correspondiente aproximación que se hace en la bibliografía (ecuaciones (18) y (19)), así como con el caso en el que se resuelve la ecuación diferencial (1) sin considerar ninguna aproximación en el radio de curvatura [12, 13], es conveniente definir una serie de parámetros adimensionales. Para ello se introduce el parámetro adimensional de fuerza:

$$
k=\frac{F L^{2}}{E I}
$$

las coordenadas adimensionales $u$ y $v$ :

$$
u=\frac{x}{L} \quad v=\frac{y}{L}
$$

así como los desplazamientos adimensionales horizontal y vertical del extremo libre de la barra, $u_{f}$ y $v_{f}$, respectivamente, definidos mediante las ecuaciones:

$$
u_{f}=\frac{\delta_{x}}{L} \quad v_{f}=\frac{\delta_{y}}{L}
$$

Haciendo uso de las ecuaciones (20)-(22), las ecuaciones $(10),(11)$ y (17) pueden escribirse en la forma:

$$
\begin{gathered}
v=\frac{k}{6}\left[3\left(1-u_{f}\right) u^{2}-u^{3}\right] \\
v_{f}=\frac{k}{3}\left(1-u_{f}\right)^{3} \\
\int_{0}^{1-u_{f}} \sqrt{1+\frac{k^{2}}{4}\left[2\left(1-u_{f}\right) u-u^{2}\right]^{2}} d u=1
\end{gathered}
$$

Asimismo, la ecuación (13) de validez de la aproximación para pequeñas pendientes se escribirá ahora:

$$
\frac{k^{2}}{4}\left(1-u_{f}\right)^{4}<<1
$$


La ecuación (25) permite obtener $u_{f}$ en función del parámetro adimensional $k$. Sin embargo, la incógnita $u_{f}$ aparece tanto en el integrando como en uno de los límites de integración. Para obtener el valor de $u_{f}$ se propone seguir un procedimiento de ensayo-error muy interesante e instructivo para los estudiantes. En primer lugar se define una función $g$ de la variable $u_{f}$ de la siguiente forma:

$$
g\left(u_{f}\right)=\int_{0}^{1-u_{f}} \sqrt{1+\frac{k^{2}}{4}\left[2\left(1-u_{f}\right) u-u^{2}\right]^{2}} d u-1
$$

con ayuda de esta función, la ecuación (25) puede escribirse como:

$$
g\left(u_{f}\right)=0
$$

En este momento se hace uso del hecho de que los estudiantes universitarios suelen estar familiarizados con software como el Mathematica, del que existe además una versión para estudiantes. Por esta razón, la integral de la ecuación (27), para cada valor de $k$, puede resolverse utilizando el comando de integración numérica "NIntegrate" del programa Mathematica. La Fig. 4 muestra los resultados obtenidos para el caso $k=0.5$. Como puede verse existe una solución de la ecuación (28) que se determina mediante un procedimiento de ensayo-error variando el valor de $u_{f}$ hasta que $g\left(u_{f}\right)$ sea menor que $10^{-8}$, que se toma como 0 a efectos computacionales.

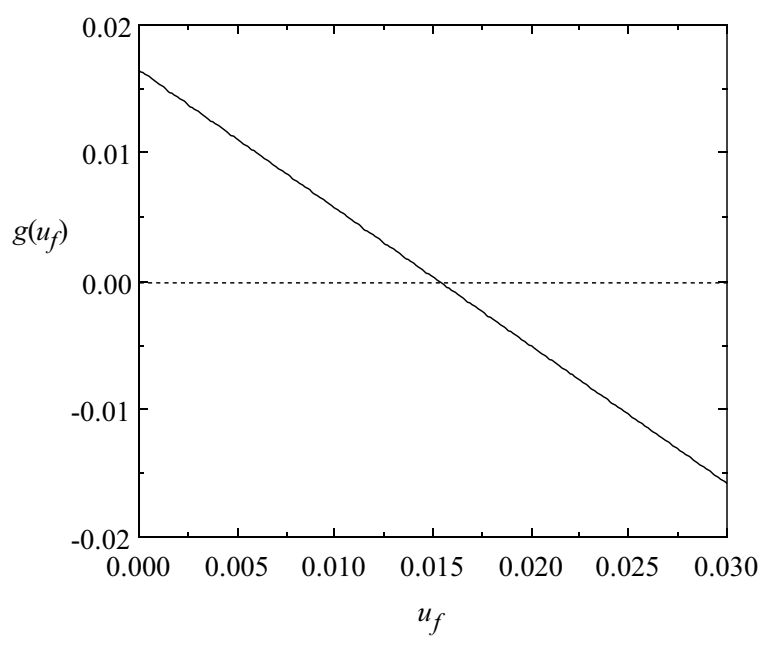

Figura 4. Función $g\left(u_{f}\right)$ para $k=0.5$.

La Fig. 5 muestra los resultados obtenidos de $u_{f}$ en función de $k$ comparados con los exactos calculados sin hacer ninguna aproximación, resolviendo las integrales elípticas que aparecen en este caso (ver Apéndice I) [12, 13]. Es importante señalar que en la aproximación que se hace en todos los libros universitarios que abordan el problema de la flexión de una barra para el caso de pequeñas pendientes se toma como hipótesis de partida $u_{f}=0$, ya que se suponde que la fuerza $F$ está aplicada siempre en el punto de abcisa $x=L$. En la Fig. 6 se han representado los errores relativos que se cometen cuando se hace uso de la aproximación que se ha considerado en este trabajo y la que se hace en la bibliografía, respecto a los valores exactos que se obtienen cuando no se hace ninguna aproximación [12]. Para la aproximación considerada en este trabajo el error relativo está por debajo del $10 \%$ para $k<0.8$, mientras que con la aproximación que aparece en todos los libros el error relativo es del $100 \%$.

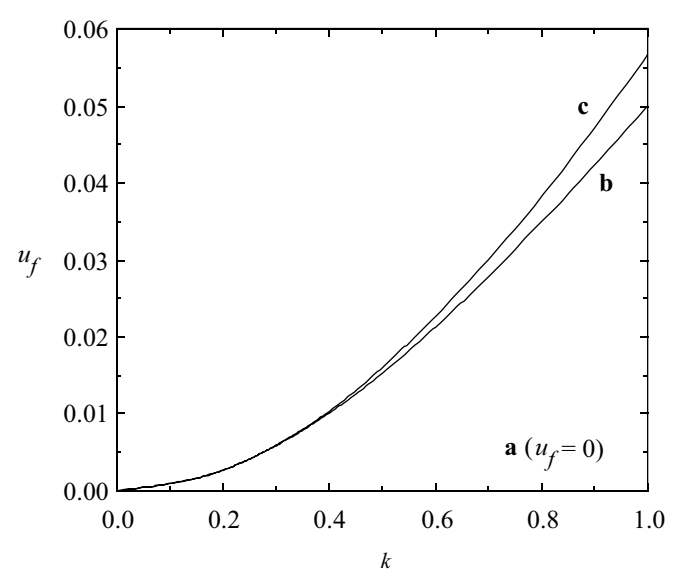

Figura 5. Valores de los desplazamientos horizontales adimensionales del extremo libre de la barra en función de $k$ : Aproximación para pequeñas pendientes realizada en la bibliografía (a), aproximación realizada en este trabajo (b) y valores exactos (c).

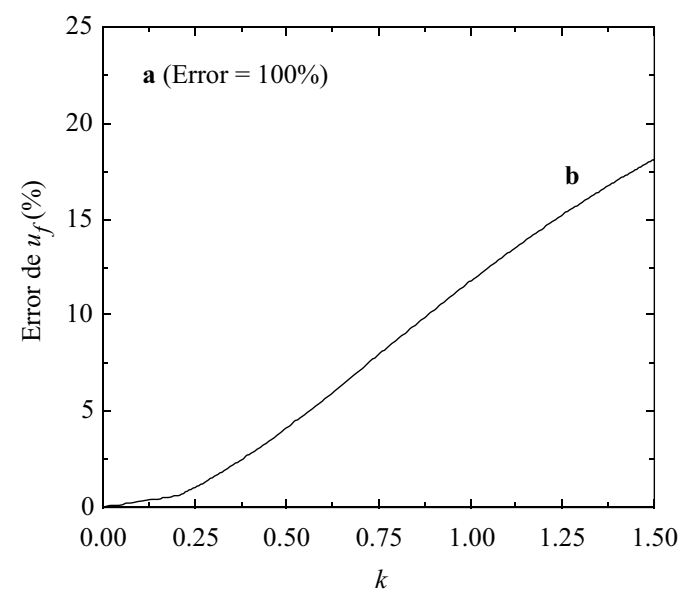

Figura 6. Errores relativos de los desplazamientos horizontales adimensionales del extremo libre de la barra en función de $k$ : Aproximación para pequeñas pendientes realizada en la bibliografía (a) y aproximación realizada en este trabajo (b).

Una vez determinado el valor de $u_{f}$ para cada $k$ es posible calcular el desplazamiento vertical adimensional del extremo libre de la barra, $v_{f}$, sin más que utilizar la ecuación (23). La Fig. 7 muestra los resultados obtenidos comparados con los exactos [12] y con los aproximados para pequeñas pendientes que se obtienen 
utilizando la ecuación (19) que se muestra en todos los libros y que ahora se escribirá en la forma $v_{f}=k / 3$. En la Fig. 8 se han representado los errores relativos correspondondientes a ambos casos. Como puede verse de las Figs 7 y 8, la aproximación para pequeñas pendientes que se ha presentado en este trabajo proporciona resultados más acordes con la realidad representada por la solución exacta para un rango de valores de $k$ mayor, pues el error relativo está por debajo del $10 \%$ para las $k$ consideradas, mientras que con la aproximación que aparece en los libros este error es mucho mayor. En la Fig. 9 se ha representado el cuadrado de la derivada primera de la función $v$ evaluado en el extremo libre de la barra, es decir, en el punto de abcisa $u=1-u_{f}$.

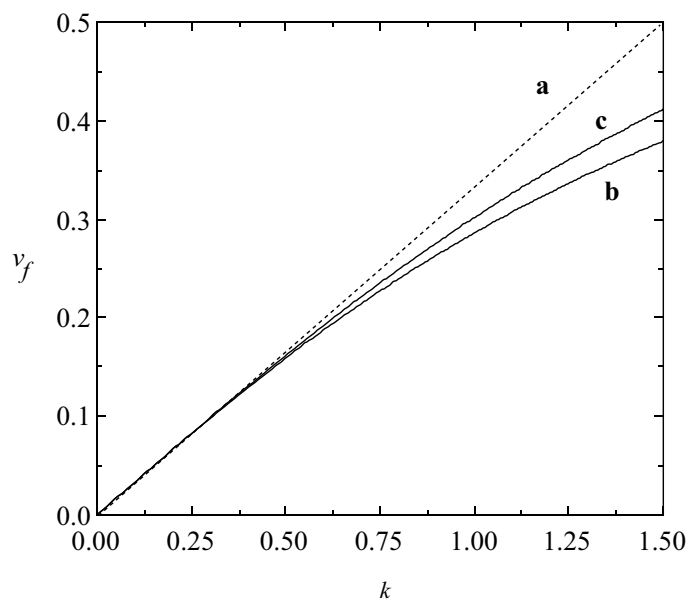

Figura 7. Valores de los desplazamientos verticales adimensionales del extremo libre de la barra en función de $k$ : Aproximación para pequeñas pendientes realizada en la bibliografía (a), aproximación realizada en este trabajo (b) y valores exactos (c).

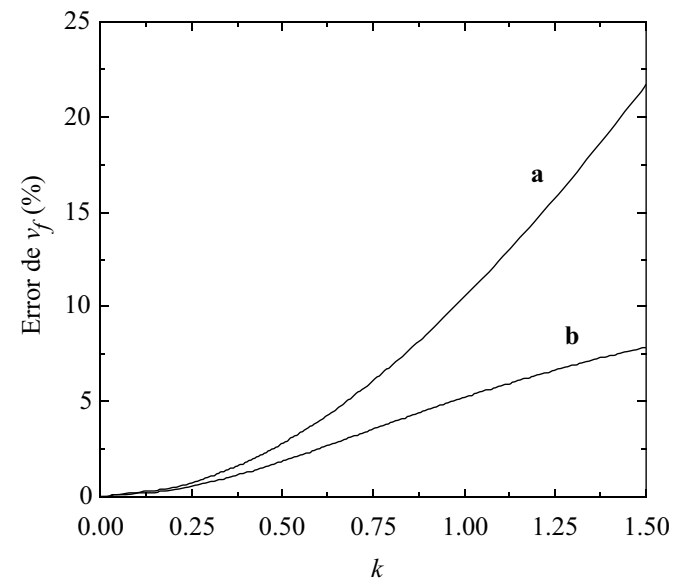

Figura 8. Errores relativos de los desplazamientos verticales adimensionales del extremo libre de la barra en función de $k$ : Aproximación para pequeñas pendientes realizada en la bibliografía (a) y aproximación realizada en este trabajo (b).

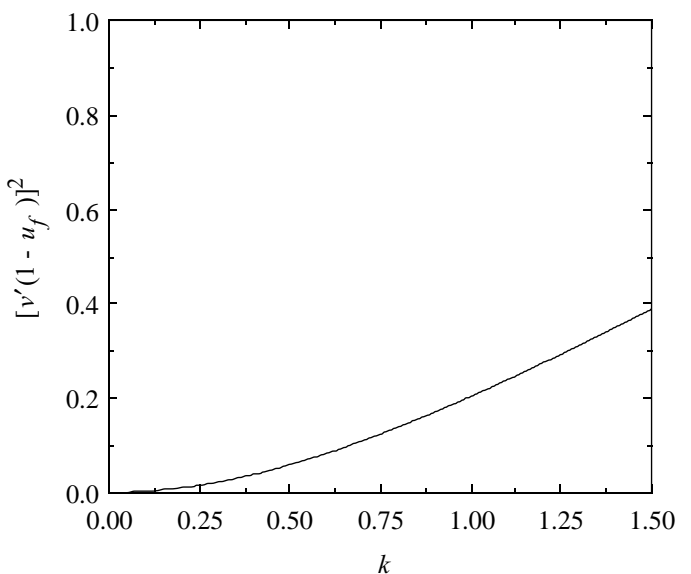

Figura 9. Cuadrado de la derivada primera de la función $v$ evaluado en el extremo libre de la barra, es decir, en el punto de abcisa $u=1-u_{f}$.

Finalmente, en la Fig. 10 se han representado las elásticas que se obtienen utilizando la ecuación (23) para $k=0.2,0.4,0.6$ y 0.8 . Como puede verse, tanto $u_{f}$ como $v_{f}$ son distintos de 0 .

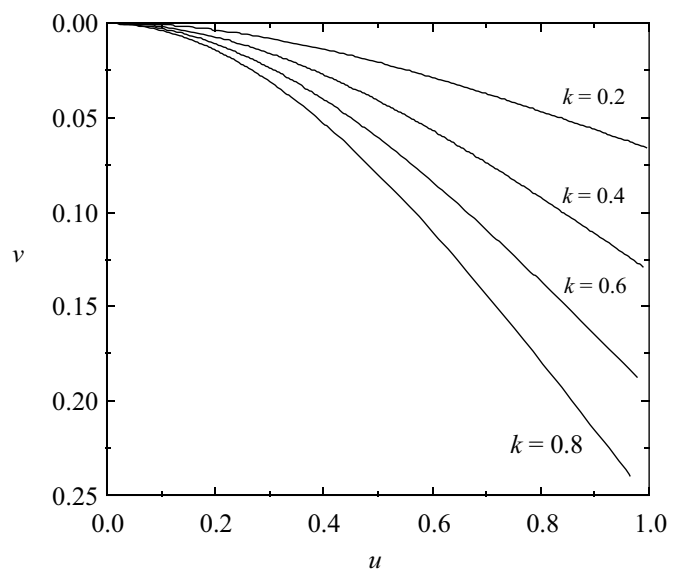

Figura 10. Elásticas adimensionales para distintos valores de $k$ obtenidas con la aproximación para pequeñas pendientes presentada en este trabajo.

\section{Resultados experimentales}

La Fig. 11 muestra una fotografía de la barra empotrada por un extremo y sobre la que se aplica una fuerza vertical concentrada en el extremo libre. La barra es una regla de acero de longitud $L$ cuya sección transversal es rectangular de base $b$ y altura $h$. La regla se ha empotrado con ayuda de una doble nuez de las que se encuentran en todos los laboratorios de Física, la cual se ha sujetado a una varilla vertical situada sobre un soporte. Con ayuda de dos láminas rectangulares metálicas colocadas una arriba de la regla y la otra debajo se ajusta la regla a la doble nuez tal y como se ve en la Fig. 12. Los valores de $b$ y $h$ de la sección rectangular de la barra delgada se han determinado con 
ayuda de un pie de rey y un palmer, respectivamente. Se han tomado medidas de $b$ y $h$ cada $5 \mathrm{~cm}$ a lo largo de la regla y se ha calculado la media de cada una de la series de medidas. Como error absoluto de $b$ y $h$ se ha tomado el valor más grande de la sensibilidad del aparato de medida y la desviación media de las medidas realizadas, siendo en ambos casos mayor la sensibilidad de los instrumentos de medida utilizados. El momento de inercia de la sección rectangular lo calculamos mediante la ecuación [1]:

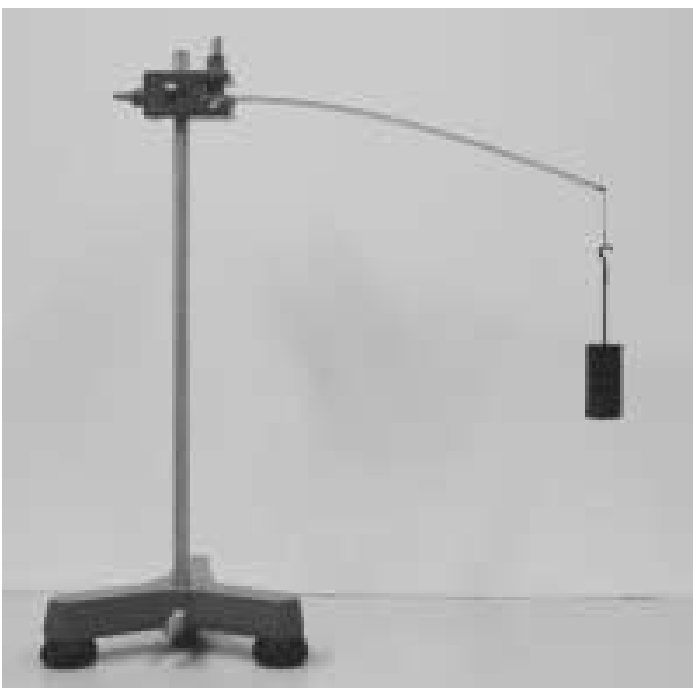

Figura 11. Fotografía de la regla de acero empotrada en un extremo analizada experimentalmente en la que se aprecia la curva elástica de la barra flexionada.

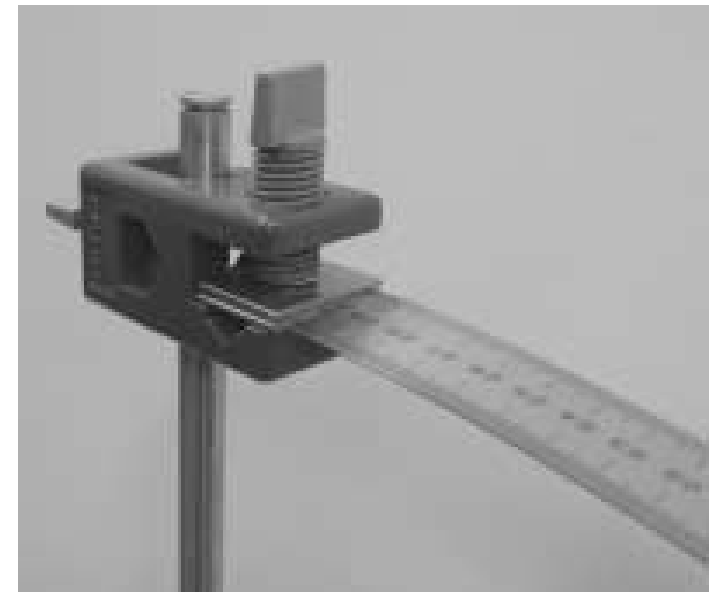

Figura 12. Fotografía del detalle del empotramiento de la regla de acero analizada experimentalmente.

$$
I=\frac{1}{12} b h^{3}
$$

Con una balanza electrónica se determina la masa de la regla y tomando $g=9.80 \pm 0.01 \mathrm{~m} / \mathrm{s}^{2}$ se calcula su peso total y, a partir de éste, el peso $P$ para una longitud de la regla de $30 \mathrm{~cm}$. En la Tabla I se indican las características de la barra delgada analizada.
TABLA I

\begin{tabular}{|c|c|}
\hline $\mathrm{L}$ & $0.3000 \pm 0.0005 \mathrm{~m}$ \\
\hline$b$ & $0.03040 \pm 0.00002 \mathrm{~m}$ \\
\hline$h$ & $0.00078 \pm 0.00001 \mathrm{~m}$ \\
\hline $\mathrm{I}$ & $(1.20 \pm 0.05) \times 10^{-12} \mathrm{~m}^{4}$ \\
\hline $\mathrm{P}$ & $0.554 \pm 0.008 \mathrm{~N}$ \\
\hline
\end{tabular}

A continuación se ha medido el desplazamiento vertical máximo del extremo libre de la barra cuando se somete a distintas fuerzas concentradas $F$ tomando como valor de referencia la posición del extremo libre cuando no se aplica ninguna fuerza $F$ (Fig. 13). Como se va a analizar la situación de pequeñas pendientes, la ecuación diferencial que hay que considerar es lineal lo que implica que el desplazamiento vertical total del extremo libre de la barra será la suma de los desplazamientos debidos a la fuerza aplicada y al peso propio. Cuando el desplazamiento vertical máximo debibo únicamente al peso propio de la barra es muy pequeño (en el laboratorio se ha medido experimentalmente que este desplazamiento vertical es menor de $8 \mathrm{~mm}$ ) y además las pendientes al aplicar fuerzas externas son pequeñas, se puede suponer, sin cometer un error significativo, que el desplazamiento del extremo libre de la barra debido a su peso propio es independiente de la fuerza aplicada. En la Fig. 14 se han representado los valores experimentales de los desplazamientos verticales $\delta_{y}$ medidos respecto a la posición del extremo libre en ausencia de fuerza aplicada, en función de la fuerza $F$. Como puede verse para fuerzas aplicadas pequeñas la relación entre $\delta_{y}$ y $F$ es lineal, pero conforme aumenta el valor de la fuerza aplicada, la relación entre $\delta_{y}$ y $F$ ya no es lineal. Analizando la zona de comportamiento lineal, que se puede tomar para fuerzas aplicadas entre 0 y 1.2 $\mathrm{N}$ (Fig. 15), se ha determinado el valor de la rigidez de la barra mediante un ajuste de mínimos cuadrados de la ecuación (19), obteniéndose:

$$
E I=0.238 \pm 0.009 \mathrm{~N} \mathrm{~m}^{2}
$$

Lo que implica que el módulo de Young del acero del que está fabricada la regla es $E=198 \pm 15 \mathrm{GPa}$. Este valor concuerda con el módulo de Young del acero que se puede encontrar en la bibliografía y que está en torno a $200 \mathrm{GPa}[14]$. En la Fig. 14 se han representado, junto con las medidas experimentales de $\delta_{y}$, los valores calculados teóricamente haciendo uso de las aproximaciones para pequeñas pendientes de la bibliografía (ecuación (19)) y la que se ha considerado en este trabajo (ecuación (11)). 

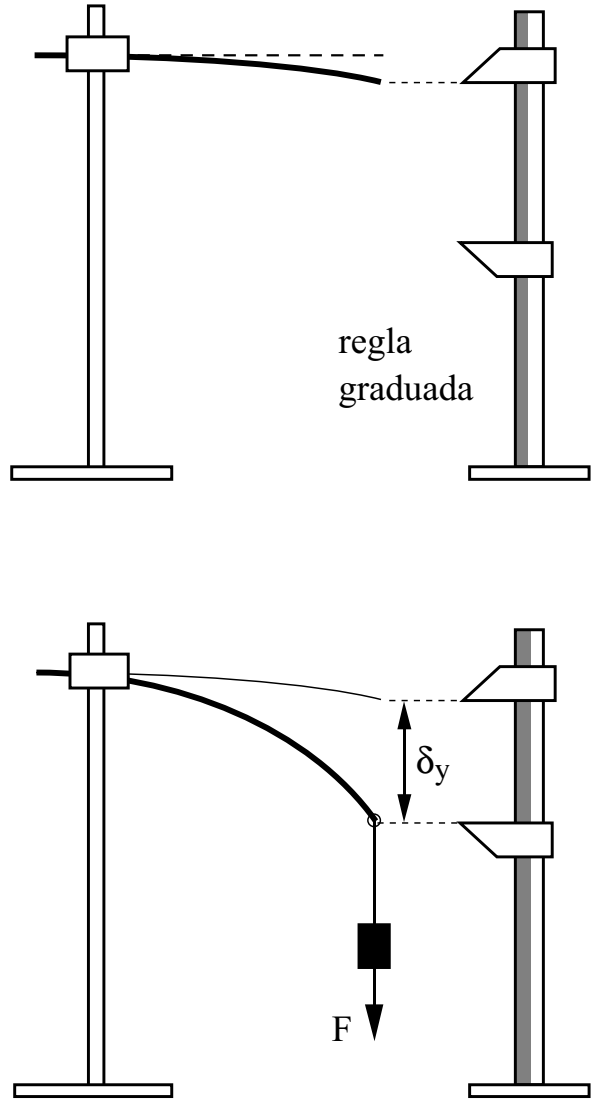

Figura 13. Determinación experimental del desplazamiento vertical del extremo libre de la barra flexionada debido a la fuerza exterior $F$ aplicada.

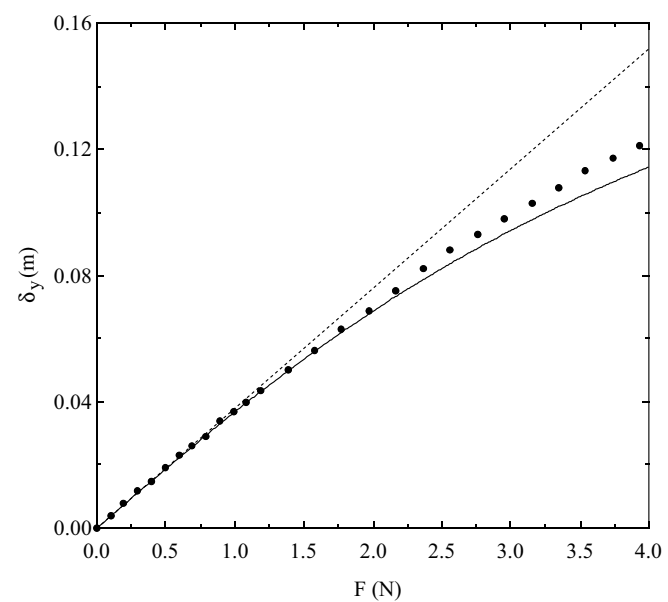

Figura 14. Valores experimentales de los desplazamientos verticales del extremo libre de la barra en función de la fuerza aplicada $F$ : Aproximación para pequeñas pendientes realizada en la bibliografía (línea recta) y aproximación presentada en este trabajo (línea curva).

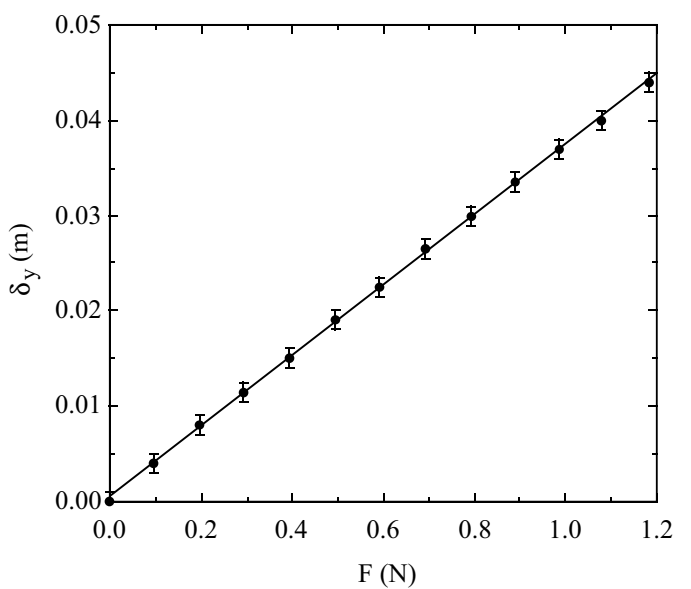

Figura 15. Valores experimentales de los desplazamientos verticales del extremo libre de la barra en función de $F$ para la zona lineal de la Fig. 14 y recta de ajuste de la ecuación (19).

\section{Conclusiones}

Partiendo de la hipótesis de que el material del que está fabricada una barra delgada flexionada es elástico lineal, se ha presentado la ecuación diferencial de la elástica de la barra de forma análoga a como se hace, por ejemplo, en el libro de Feynman [5] y en otros libros $[2,4,7,8]$. Esta ecuación diferencial se ha simplificado considerando el caso de pequeños desplazamientos de la directriz de la barra (pendientes pequeñas), pero sin hacer una aproximación tan extrema a como se hace en los textos universitarios de Física y Mecánica. Un aspecto importante y de gran interés didáctico es la forma en como se ha aplicado la condición de que la barra es inextensible (la longitud de su fibra neutra no cambia), aspecto que no se considera en los libros antes mencionados. Se han obtenido las ecuaciones de la elástica y los desplazamientos vertical y horizontal del extremo libre de la barra, comparándolos con los correspondientes al caso general de pequeñas y fuertes pendientes, asi como con la aproximación de pequeñas pendientes que aparece en la bibliografía. En este último caso se supone que el desplazamiento horizontal del extremo libre de la barra es nulo. Los resultados teóricos se han comparado con los experimentales obtenidos en el laboratorio con una regla de acero, comprobándose como la aproximación para pequeñas pendientes considerada en este trabajo es "menos aproximada" que la que aparece en la bibliografía. Por último, y no menos importante, en el desarrollo del trabajo se han utilizado conceptos físicos de gran interés en un curso de Física o Mecánica (esfuerzo, momento flector, fuerza puntual, módulo de Young, elástica, momento de inercia de una sección plana, flexión, deformación, etc.), así como de cálculo infinitesimal (derivada, integral, radio de curvatura de una curva, etc.), junto con la utilización de software 
como el Mathematica para resolver numéricamente integrales.

\section{Apéndice I: Elástica de una barra delgada en voladizo para el caso general}

En el caso general en el que los desplazamientos de la barra delgada en voladizo son grandes, lo que implica fuertes pendientes de la curva elástica, no es posible hacer uso de la aproximación de la ecuación (3) para el radio de curvatura. En esta situación, en vez de utilizar la ecuación exacta (2) para el radio de curvatura en coordenadas cartesianas, es mejor resolver el problema en función de la longitud de $\operatorname{arco} s$ a lo largo de la elástica de la barra y del ángulo $\varphi$, que es el ángulo que forma con la horizontal la recta tangente a la curva elástica en un punto determinado de la misma (ver Fig. 2 ). Procediendo de esta forma se obtiene una ecuación diferencial no lineal de segundo orden cuya solución se expresa en términos de integrales elípticas [12, 13]. Se dice que el problema presenta no linealidad geométrica. Para la longitud $L$ de la barra se obtiene la ecuación [12]:

$$
L=\sqrt{\frac{E I}{2 F}} \int_{0}^{\varphi_{0}} \frac{d \varphi}{\sqrt{\sin \varphi_{0}-\sin \varphi}}
$$

Esta ecuación permite determinar el ángulo $\varphi_{0}$ en el extremo libre de la barra (Fig. 2) en función de la longitud de la barra, $L$, el momento de inercia de su sección transversal, $I$, el módulo de Young del material, $E$, y la fuerza aplicada, $F$. Asimismo, las coordenas $(x, y)$ de los puntos de la curva elástica de la barra delgada en voladizo pueden calcularse en función del ángulo $\varphi$ mediante las ecuaciones [12]:

$$
\begin{gathered}
x(\varphi)=\sqrt{\frac{2 E I}{F}}\left(\sqrt{\sin \varphi_{0}}-\sqrt{\sin \varphi_{0}-\sin \varphi}\right) \\
y(\varphi)=\sqrt{\frac{E I}{2 F}} \int_{0}^{\varphi} \frac{\sin \varphi d \varphi}{\sqrt{\sin \varphi_{0}-\sin \varphi}}
\end{gathered}
$$

Por último, los desplazamientos vertical y horizontal del extremo libre de la barra se calculan tomando $\varphi$ $=\varphi_{0}$ en las ecuaciones (A7) y (A8):

$$
\begin{gathered}
\delta_{x}=L-x\left(\varphi_{0}\right) \\
\delta_{y}=y\left(\varphi_{0}\right)
\end{gathered}
$$

Las integrales elípticas de las ecuaciones (A1) y (A3) pueden expresarse en términos de funciones elípticas, sin embargo, y tal y como se ha señalado en una publicación reciente [12] o se hace, por ejemplo, en un problema similar a éste como es el péndulo simple para grandes oscilaciones [15], para los estudiantes de los primeros cursos universitarios es más ilustrativo y didácticamente más aconsejable que las resuelvan con ayuda de algún programa de ordenador como el Mathematica.

\section{Referências}

[1] W. F. Riley y L. D. Sturges, Ingeniería Mecánica: Estática (Reverté, Barcelona, 1995).

[2] F. Belmar, A. Garmendía y J. Llinares, Curso de Física Aplicada: Estática (Universidad Politécnica de Valencia, 1987).

[3] J. L. Huertas y S. Val, Mecánica (20 de Bachillerato) (McGraw-Hill, Madrid, 1998).

[4] M. R. Ortega, Lecciones de Física: Mecánica 3 (Edita el autor, Córdoba, 1987).

[5] R. Feynman, R. B. Leighton y M. Sands, Física, Vol. II: Electromagnetismo y Materia (Addison-Wesley Iberoamericana, México, 1987).

[6] A. Bedford y W. Fowler, Mecánica para Ingeniería: Estática (Addison-Wesley Iberoamericana, Wilmington, 1987)

[7] L. Ortiz-Berrocal, Resistencia de Materiales (McGrawHill, Madrid, 1997).

[8] R. C. Hibbeler, Mecánica de Materiales (Prentice Hall, México, 1998).

[9] F. R. Zypman y C. Guerra-Vela, "The macroscopic scanning force 'microscope"', Eur. J. Phys. 22, 17-30 (2001).

[10] Th. Höpfl, D. Sander y J. Kirschener, "Demonstration of different bending profiles of a cantilever caused by a torque or a force", Am. J. Phys. 69, 1113-1115 (2001).

[11] A. Beléndez, C. Neipp y T. Beléndez, "Estudio experimental de una viga en voladizo", Rev. Esp. Fis. 15 (3) 42 (2001).

[12] T. Beléndez, C. Neipp y A. Beléndez, "Large and small deflections of a cantilever beam", Eur. J. Phys. 23, 371-379 (2002).

[13] L. D. Landau y E. M. Lifshitz, Course of Theoretical Physics, Vol. 7: Theory of Elasticity (Pergamon Press, Oxford, 1986).

[14] P. A. Tipler, Física para la Ciencia y la Tecnología, Vol. 1 (Reverté, Barcelona, 1999).

[15] T. F. Zheng, M. Mears, D. Hall y D. Pushkin, "Teaching the nonlinear pendulum", Phys. Teach. 32, April, 248-251 (1994). 